Pacific Journal of Mathematic 


\title{
COLLECTIONS AND SEQUENCES OF CONTINUA IN THE PLANE
}

\author{
C. E. Burgess
}

1. An inversion of the plane with respect to a closed circular disc. The inversion described here will be used in proving Theorem 5 .

Definition. Let $S$ denote the plane, let $K$ be a closed circular disc, and let $T$ be a one-to-one transformation of $S$ onto itself satisfying the following conditions:

(1) $T$ is continuous over $K$, and $T(K)=K$;

(2) $T$ is continuous over $S-K$, and $T(S-K)=S-K$; and

(3) if $H$ is an unbounded subset of $S-K$ which does not have a limit point in $K$, then $T(H)$ is bounded and has a limit point in $K$.

The transformation $T$ will be called an inversion of $S$ with respect to $K$.

Notation. If $T$ is an inversion of the plane with respect to a closed circular disc $K$, and $M$ is a continuum in $S-K$, then $M^{\circ}$ will denote the closure of $T(M)$. If $G$ is a collection of continua in $S-K$, then $G^{\prime}$ will denote the collection of all continua $X^{\prime}$ such that $X$ is a continuum of $G$. This notation will be used in the statement of Theorem 1 and in the proof of Theorem 5 .

THE OREM 1. If $K$ is a closed circular disc and $G$ is a finite collection of mutually exclusive unbounded continua not intersecting $K$, then there is an inversion $T$ of the plane with respect to $K$ such that the continua of $G^{\prime}$ are bounded and mutually exclusive. ${ }^{1}$

Indication of proof. If the plane $S$ is inverted about the boundary of $K$ with respect to the center $o$ of $K$, then the continua of $G$ are carried onto mutually exclusive bounded connected sets each of which has $o$ as a limit point and is closed relative to $S-o$. Hence it will be sufficient to show that if $M_{1}, M_{2}, \cdots$, $M_{n}(n>1)$ are bounded continua such that

\footnotetext{
${ }^{1}$ I am indebted to the referee for some very helpful suggestions which enabled me to obtain a simplified proof of this theorem.

Received September 29, 1953. Presented to the American Mathematical Society, May 2, 1953.
}

Pacific J. Math. 5 (1955), $325-333$ 


$$
M_{i} \cdot M_{j}=o \text { for each } i \text { and } j(i, j \leq n)(i \neq j) \text {, }
$$

and $M_{i}-o$ is connected, then there is a homeomorphism $Z$ of $S-o$ onto $S-K$ such that the closures of

$$
Z\left(M_{1}-o\right), Z\left(M_{2}-o\right), \cdots, Z\left(M_{n}-o\right)
$$

are mutually exclusive bounded continua. By using a theorem proved by Lubben [4, Theorem 18], it can be shown that there exist $n-1$ simple closed curves $J_{1}, J_{2}, \ldots, J_{n-1}$ such that

(1) $J_{i} \cdot J_{j}=o$ for each $i$ and $j(i, j<n)(i \neq j)$ and

(2) each of the $n$ complementary domains of $J_{1}+J_{2}+\cdots+J_{n-1}$ contains one of the sets $M_{1}-o, M_{2}-o, \cdots, M_{n}-o$. Furthermore, it can be shown that there exist $n-1$ simple closed curves $H_{1}, H_{2}, \cdots, H_{n-1}$ satisfying the conditions required above for $J_{1}, J_{2}, \cdots, J_{n-1}$ such that $J_{i} \cdot H_{j}=o$ for each $i$ and $j(i$, $j<n)$, and the connected domain having $J_{i}+H_{i}$ as its boundary does not intersect $M_{1}+M_{2}+\cdots+M_{n}$. There is a home omorphism $T_{1}$ of $S$ onto itself leaving $o$ fixed such that for each $i(i<n), T_{1}\left(J_{i}\right)$ and $T_{1}\left(H_{i}\right)$ are polygons. There is no loss of generality in assuming that $K$ has radius 1 and that $T_{1}(K)=K$. Consider a polar coordinate system with origin $o$, and let $T_{2}$ be a transformation that carries $(\rho, \theta)$ into $(\rho+1, \theta)$, where $\rho>0$. Then $T_{2} T_{1}$ is the desired homeomorphism $Z$.

\section{Some properties of continua with respect to point sets which intersect} them.

Notation and definitions. If $G$ is a collection of point sets, then $G^{*}$ denotes the sum of the sets of $G$.

If $G$ is a collection of point sets and $M$ is a continuum intersecting every set of $G$ such that no proper subcontinuum of $M$ intersects every set of $G$, then $M$ is said to be irreducible among the sets of $G$.

If $G$ is a collection of mutually exclusive closed point sets and $M$ is a continuum intersecting every set of $G$ such that for some two sets $K_{1}$ and $K_{2}$ of $G$ the set $M-M \cdot\left(K_{1}+K_{2}\right)$ is connected, then $M$ is said to have property $X$ with respect to $G$.

If $p$ is a point of the continuum $M$, the sum of all proper subcontinua of $M$ containing $p$ is called a composant of $M$.

A proper subset $H$ of the continuum $M$ is said to be a set of condensation of $M$ if every point of $H$ is a limit point of $M-H$. 
THEOREM 2. If $M$ is a compact indecomposable metric continuum and $G$ is a countable collection of closed subsets of $M$ such that no set of $G$ intersects every composant of $M$, then uncountably many composants of $M$ lie in $M-G^{*}$.

\section{LEMma 2.1. If}

(1) $M$ is a compact indecomposable metric continuum,

(2) $H$ is a closed subset of $M$ such that some composant $K$ of $M$ does not intersect $H$,

(3) $D$ is a domain intersecting $K$ but not $H$, and

(4) $W$ is the collection of all components of $M-M \cdot D$ that intersect $H$, then $\mathbb{W}^{*}$ is closed and each of its points is a limit point of $M-W^{*}$.

Proof of Lemma 2.1. Suppose that $W^{*}$ is not closed. Since $W^{*}$ contains $H$, then some point $p$ of $M-\left(D+H+W^{*}\right)$ is a limit point of $\mathbb{W}^{*}$. There exists a sequence of continua $L_{1}, L_{2}, L_{3}, \cdots$ converging to a continuum $L$ containing $p$ such that for each $n, L_{n}$ is an element of $W$. Since each $L_{n}$ intersects the closed set $H$, then $L$ intersects $H$. This means that $L$ is a subset of some element of $W$. Hence $W^{*}$ is closed. Since each element of $W$ intersects $H$ and is a proper subcontinuum of the indecomposable continuum $M$, then $W^{*}$ does not intersect $K$. Since $K$ is dense in $M$, then every point of $W^{*}$ is a limit point of $M-W^{*}$.

Proof of Theorem 2. Let $X$ be an element of $G$. By applying Lemma 2.1 to each element of a sequence of domains in $M-X$ closing down on a point of a composant of $M$ not intersecting $X$, it can be seen that the sum of all composants of $M$ intersecting $X$ is the sum of a countable number of closed sets of condensation of $M$. Since $G$ is countable, the sum of all composants of $M$ intersecting $G^{*}$ is the sum of a countable number of closed sets of condensation of $M$. Hence by Baire's theorem [6, Theorem 15, p. 11], some composant of $M$ does not intersect $G^{*}$. Since each composant of $M$ is the sum of a countable number of continua of condensation of $M$, it follows, upon again applying Baire's theorem, that uncountably may composants of $M$ lie in $M-G^{*}$.

THEOREM 3. If $G$ is a finite collection consisting of at least two mutually exclusive closed point sets and $M$ is a compact metric continuum irreducible among the sets of $G$, then there exist two sets $K_{1}$ and $K_{2}$ of $G$ such that $M-$ $M \cdot\left(K_{1}+K_{2}\right)$ is connected; that is, $M$ has property $X$ with respect to $G$.

Lemma 3.1. Suppose

(1) $G$ is a finite collection of mutually exclusive closed point sets, 
(2) $M$ is a compact metric continuum irreducible among the sets of $G$,

(3) $K_{1}, K_{2}$, and $K_{3}$ are three sets of $G$, and

(4) $M_{1}$ and $M_{2}$ are subcontinua of $M$ such that

(1) for each $i(i \leq 2), M_{i}$ intersects every set of the collection $G-K_{i}$ and does not intersect $K_{i}$,

(2) the set $M_{1}-M_{1} \cdot\left(K_{2}+K_{3}\right)$ is connected, and

(3) $M_{1}$ intersects $M_{2}$. Then $M$ has property $X$ with respect to $G$.

Proof of Lemma 3.1. There is a subcontinuum $M_{2}^{\prime}$ of $M_{2}$ irreducible from $K_{1}$ to $M_{1}$. Since the connected sets $M_{1}-M_{1} \cdot K_{2}$ and $M_{2}^{\prime}-M_{2}^{\prime} \cdot K_{1}$ have a point in common, their sum is connected. Since $M_{1}+M_{2}^{\prime}$ is a subcontinuum of $M$ intersecting every set of $G$, then $M_{1}+M_{2}^{\prime}=M$. But

$$
\begin{aligned}
\left(M_{1}-M_{1} \cdot K_{2}\right)+\left(M_{2}^{\prime}-M_{2}^{\prime} \cdot K_{1}\right) & =\left(M_{1}+M_{2}^{\prime}\right)-\left(M_{1}+M_{2}^{\prime}\right) \cdot\left(K_{1}+K_{2}\right) \\
& =M-M \cdot\left(K_{1}+K_{2}\right) .
\end{aligned}
$$

Hence $M$ has property $X$ with respect to $G$.

\section{Lemma 3.2. Theorem 3 holds true if $M$ is decomposable.}

Proof of Lemma 3.2. Suppose that for any nondegenerate proper subcollection $G_{1}$ of $G$ there is a subcontinuum of $M$ having property $X$ with respect to $G_{1}$. It will be shown that if this condition is satisfied, then $M$ has property $X$ with respect to $G$. Then Lemma 3.2 will follow by induction from the following well known facts:

(1) Theorem 3 holds true if $G$ consists of two closed sets and

(2) if $W$ is a finite collection of mutually exclusive closed sets and $K$ is a compact metric continuum intersecting every set of $W$, then some subcontinuum of $K$ is irreducible among the sets of $W$.

In addition to the above supposition, suppose that $M$ does not have property $X$ with respect to $G$. Then there exist three subcontinua $M_{1}, M_{2}$, and $M_{3}$ of $M$ and three sets $K_{1}, K_{2}$, and $K_{3}$ of $G$ such that

(1) for each $i(i \leq 3), M_{i}$ has property $X$ with respect to the collection $G-K_{i}$ and does not intersect $K_{i}$ and

(2) the set $M_{1}-M_{1} \cdot\left(K_{2}+K_{3}\right)$ is connected. By Lemma 3.1, neither of the sets $M_{2}$ and $M_{3}$ intersects $M_{1}$. Hence $M_{2}+M_{3}$ is a proper subset of $M$ 
intersecting every set of $G$. Then $M_{2}$ and $M_{3}$ have no point in common. For each $i(i \leq 3)$, let $p_{i}$ be a point of $M_{i}$. Since $M$ is decomposable, some proper subcontinuum $M^{\prime}$ of $M$ contains some two of the points $p_{1}, p_{2}$, and $p_{3}[2$, Theorem 4]. As the three cases are quite similar, consider the case in which $p_{1}+p_{2}$ is a subset of $M^{\prime}$ : Since $M^{\prime}+M_{1}+M_{2}$ is a continuum intersecting every set of $G$, then $M^{\prime}+M_{1}+M_{2}=M$. Hence $M_{3}$ is a subset of $M^{\prime}$. It follows, in the same manner, that each of the sets $M_{1}$ and $M_{2}$ is a subset of $M$ '. Then $M^{\prime}$ intersects every set of $G$. This is contradiction since $M$ is irreducible among the sets of $G$.

Proof of Theorem 3. If $M$ is decomposable, then Theorem 3 follows from Lemma 3.2. Suppose that $M$ is indecomposable. Then no composant of $M$ intersects every set of $G$. Let $L_{\mathfrak{l}}$ be a set of $G$ such that some composant of $M$ does not intersect $L_{1}$. There is a set $L_{2}$ of $G$ such that some composant of $M$ intersects $L_{1}$ but not $L_{2}$. Let $G^{\prime}$ be the collection consisting of all elements of $G$ that do not intersect every composant of $M$. By Theorem 2, some composant of $M$ lies in $M-M \cdot G^{* *}$. Since this composant is connected and dense in $M$, then $M-M \cdot G^{* *}$ is connected. As it was shown above that $G^{\prime}$ consists of at least two closed sets, then $M$ has property $X$ with respect to $G^{\prime}$. Hence $M$ also has property $X$ with respect to $G$.

THEOREM 4. If, in the plane, $K_{1}$ and $K_{2}$ are mutually exclusive bounded continua, $K_{3}$ is a closed circular disc not intersecting $K_{1}+K_{2}$, and $M_{1}, M_{2}$, and $M_{3}$ are mutually exclusive bounded continua each intersecting each of the sets $K_{1}, K_{2}$, and $K_{3}$ such that $M_{i}-M_{i} \cdot\left(K_{1}+K_{2}\right)$ is connected for each $i(i \leq 3)$, then one of the continua $M_{1}, M_{2}$, and $M_{3}$ intersects the interior of $K_{3}$.

Proof of Theorem 4. F. B. Jones [3, Theorem 28] has proved that if $J$ is the boundary of a simple domain $D$ in the plane, $H$ is connected set lying in $D+J$, and $K$ is a continuum lying in $(D+J)-H$, then no two points of $H \cdot J$ separate two points of $K \cdot J$ from each other on $J$. Repeated use of this result will be made in the following argument.

Suppose that no one of the continua $M_{1}, M_{2}$, and $M_{3}$ intersects the interior of $K_{3}$. Let $J_{3}$ denote the boundary of $K_{3}$. There exists an arc of $J_{3}$ which intersects two but not three of the continua $M_{1}, M_{2}$, and $M_{3}$. Consider the case in which such an arc intersects both $M_{2}$ and $M_{3}$. By [6, Theorem 17, p. 189], there exist two points $a_{1}$ and $a_{2}$ of $J_{3}$ and an arc $a_{1} a_{2}$ lying, except for $a_{1}$ and $a_{2}$, in the exterior of $J_{3}$ such that $a_{1}+a_{2}$ separates $J_{3} \cdot M_{1}$ from $J_{3} \cdot\left(M_{2}+M_{3}\right)$ on $J_{3}$ and $a_{1} a_{2}$ does not intersect $M_{1}+M_{2}+M_{3}$. Hence $a_{1} a_{2}$ intersects $K_{1}+K_{2}$. 
Therefore $a_{1} a_{2}$ contains two $\operatorname{arcs} a_{1} b_{1}$ and $a_{2} b_{2}$ irreducible from $J_{3}$ to $K_{1}+K_{2}$. Since the set

$$
K_{1}+a_{1} b_{1}+a_{2} b_{2}-\left(b_{1}+b_{2}\right)
$$

does not intersect the connected set

$$
K_{2}+M_{1}+M_{2}-\left(M_{1} \cdot K_{1}+M_{2} \cdot K_{1}\right)
$$

then one of the points $b_{1}$ and $b_{2}$ does not lie in $K_{1}$. Similarly $K_{2}$ does not contain both of these points. Hence one of the points $b_{1}$ and $b_{2}$ belongs to $K_{1}$ and the other belongs to $K_{2}$. It follows from [6, Theorem 17, p. 189] that there exist two points $c_{1}$ and $c_{2}$ of $J_{3}$ and an arc $c_{1} c_{2}$ lying, except for $c_{1}$ and $c_{2}$, in the exterior of $J_{3}$ such that $c_{1}$ and $c_{2}$ belong to $M_{1}$ and $M_{2}$ respectively and $c_{1} c_{2}$ does not intersect $K_{1}+K_{2}+a_{1} b_{1}+a_{2} b_{2}$. Let $H$ be an arc in $c_{1} c_{2}$ irreducible from $M_{1}$ to $M_{2}$. Since $a_{1}+a_{2}$ separates $J_{3} \cdot M_{1}$ from $J_{3} \cdot M_{2}$ on $J_{3}$, then the connected set

$$
H+M_{1}+M_{2}-\left(M_{1}+M_{2}\right) \cdot\left(K_{1}+K_{2}\right)
$$

intersects the continuum

$$
K_{1}+K_{2}+M_{3}+a_{1} b_{1}+a_{2} b_{2} \text {. }
$$

Hence $H$ intersects $M_{3}$. Then there is an arc $H^{\prime}$ in $H$ intersecting both $M_{1}$ and $M_{3}$ but not $M_{2}$. The connected set

$$
H^{\prime}+M_{1}+M_{3}-\left(M_{1}+M_{3}\right) \cdot\left(K_{1}+K_{2}\right)
$$

does not intersect the continuum

$$
K_{1}+K_{2}+M_{2}+a_{1} b_{1}+a_{2} b_{2} \text {. }
$$

This is contrary to [3, Theorem 28].

THEOREM 5. If, in the plane, $\mathbb{W}$ is a collection consisting of $n$ mutually exclusive bounded continua and $G$ is a collection consisting of $n^{2}-n+1$ mutually exclusive continua each intersecting every continuum of $W$, then some continuum of $G$ contains a bounded continuum which intersects every continuum of $\mathbb{W}$.

Proof of Theorem 5. Use the notation described in $\S 1$. Let $K$ be a closed 
circular disc not intersecting $G^{*}+W^{*}$. By Theorem 1 , there is an inversion $T$ of the plane with respect to $K$ such that the continua of $G^{\prime}$ are bounded and mutually exclusive and no one of them intersects the interior of $K$. The continua of $W^{\prime}$ are mutually exclusive and bounded and no one of them intersects $K$. Since Theorem 5 obviously holds true if $n=1$, suppose that $W^{\prime}$ consists of at least two continua. Each continuum of $G^{\prime}$ contains a continuum irreducible among the sets of $\mathbb{W}^{\prime}$. Hence by Theorem 3, each continuum of $G^{\prime}$ contains a continuum which has property $X$ with respect to $W^{\prime}$. Since the number of continua of $G^{\prime}$ is one more than twice the number of distinct pairs of elements of elements of $W^{\prime}$, then there exist three mutually exclusive continua $M_{1}^{\prime}, M_{2}^{\prime}$, and $M_{3}^{\prime}$ and two continua $K_{1}^{\prime}$ and $K_{2}^{\prime}$ of $W^{\prime}$ such that

(1) for each $i(i \leq 3), M_{i}^{\prime}$ is a subset of a continuum of $G^{\prime}$ and intersects every continuum of $\mathbb{W}^{\circ}$ and

(2) $M_{i}^{\prime}-M_{i}^{\prime} \cdot\left(K_{1}^{\prime}+K_{2}^{\prime}\right)$ is connected. Since no one of the continua $M_{1}^{\prime}$, $M_{2}^{\prime}, M_{3}^{\prime}$ intersects the interior of $K$, it follows from Theorem 4 that some $M_{j}^{\prime}$ $(j \leq 3)$ does not intersect $K$. Then $T^{-1}\left(M_{j}^{\prime}\right)$ is a bounded subcontinuum of some continuum of $G$ and intersects every continuum of $W$.

3. Convergent sequences of continua. The author has previously shown that Theorem 6 holds true if $M_{1}, M_{2}, M_{3}, \ldots$ are compactly connected [ 1 , Theorem $5]$. As a consequence of Theorem 6 , the requirement in the hypotheses of Theorems 7 and 8 of [1] that the continua of $\alpha$ be compactly connected can be omitted.

THEOREM 6. If $M_{1}, M_{2}, M_{3}, \cdots$ is a sequence of mutually exclusive nondegenerate continua in the plane converging to a continuum $M$, then there is a sequence $T_{1}, T_{2}, T_{3}, \ldots$ of bounded continua converging to $M$ such that for each $n, T_{n}$ is a subset of $M_{n}$ and is irreducible between some two points.

With the aid of Theorem 5, this theorem can be proved by an argument quite similar to the argument given to prove Theorem 5 of [1].

4. A property of a certain type of unbounded continuum. R. L. Moore [5] has shown that the plane does not contain uncountably many mutually exclusive triodic continua. N.E. Rutt [8] has shown that if $M$ is a continuum containing two unbounded continua neither of which contains the other, and $M$ does not separate the plane, then the plane does not contain uncountably many mutually exclusive continua such as $M$. There exist continua which satisfy both the hypothesis of Rutt's theorem and the hypothesis of Theorem 7. However, in 
Theorem 7 it is not required that $M$ should not separate the plane. It should also be noted that there exist continua which satisfy the hypothesis of Rutt's theorem but do not satisfy the hypothesis of Theorem 7. (See [7, Example 1].)

THEOREM 7. Let $M$ be a continuum having the property that for some positive integer $n$ there exist $n$ domains intersecting $M$ such that no bounded subcontinuum of $M$ intersects each of these domains. The plane does not contain uncountably many mutually exclusive continua such as $M$.

Proof of Theorem 7. Suppose that there exists a collection $G$ consisting of uncountably many mutually exclusive such continua in the plane. There exist an uncountable subcollection $G_{1}$ of $G$ and a positive integer $r$ such that if $Y$ is a continuum of $G_{1}$ then there exist $r$ domains each intersecting $Y$ such that no bounded subcontinuum of $Y$ intersects each of these domains. Then there exist a sequence $M_{1}, M_{2}, M_{3}, \cdots$ of distinct continua of $G_{1}$ and a positive number $d$ such that

(1) for each $i$, there exist $r$ points $p_{i 1}, p_{i 2}, \cdots, p_{i r}$ of $M_{i}$ and $r$ mutually exclusive circular domains $D_{i 1}, D_{i 2}, \cdots, D_{i r}$ having the points $p_{i 1}, p_{i 2}, \cdots$, $p_{i r}$ respectively as centers and having a diameter greater than $d$,

(2) for each $j(j \leq r)$, the sequence $p_{1 j}, p_{2 j}, p_{3 j}, \cdots$ converges to a point $p_{j}$, and

(3) for each $i$, no bounded subcontinuum of $M_{i}$ intersects each of the domains $D_{i 1}, D_{i 2}, \cdots, D_{i r}$. There exist mutually exclusive circular discs $K_{1}$, $K_{2}, \cdots, K_{r}$ such that for each $j(j \leq r), K_{j}$ has $p_{j}$ as its center and has a diameter less than $d / 2$. There is a positive integer $m$ such that for each $j$ $(j \leq r)$ and each $n(n>m), K_{j}$ is a subset of $D_{n j}$. Since infinitely many of the continua $M_{1}, M_{2}, M_{3}, \ldots$ intersect each of the circular discs $K_{1}, K_{2}, \cdots, K_{r}$, then by Theorem 5 , there is a positive integer $n$ greater than $m$ such that some bounded subcontinuum $H_{n}$ of $M_{n}$ intersects each of the circular discs $K_{1}, K_{2}, \cdots$, $K_{r}$. Hence $H_{n}$ also intersects each of the domains $D_{n 1}, D_{n 2}, \cdots, D_{n r}$. This involves a contradiction.

\section{REFERENCES}

1. C. E. Burgess, Continua and their complementary domains in the plane, Duke Math. J. 18 (1951), 901 - 917.

2. S. Janiszewski and C. Kuratowski, Sur les continus indécomposables, Fund. Math. 1 (1920), 210- 222.

3. F. Burton Jones, Certain consequences of the Jordan curve theorem, Amer. J. Math. 63 (1941), $531-544$. 
4. R.G. Lubben, Separation theorems with applications to questions concerning accessibility and plane continua, Trans. Amer. Math. Soc. 31 (1929), 503-522.

5. R. L. Moore, Concerning triods in the plane and the junction points of plane continua, Proc. Nat. Acad. Sci. 14 (1928), 85-88.

6. - Foundations of point set theory, Amer. Math. Soc. Colloquium Publications, 13, 1932.

7. J.H. Roberts, Concerning atriodic continua, Monatsh. Math. 37 (1930), 223-230.

8. N. E. Rutt, Some theorems on triodic continua, Amer. J. Math. 56 (1934), 122-132.

THE UNIVERSITY OF UTAH 


\section{PACIFIC JOURNAL OF MATHEMATICS}

\section{EDITORS}

\author{
H.L. ROYDEN \\ Stanford University \\ Stanford, California \\ E. HEWITT \\ University of Washington \\ Seattle 5, Washington
}

\author{
R. P. DILWORTH \\ California Institute of Technology \\ Pasadena 4, California \\ * Alfred Horn \\ University of California \\ Los Angeles 24, California
}

\section{ASSOCIATE EDITORS}

\section{H, BUSEMANN \\ HERBERT FEDERER}

MARSHALL HALL

\section{P.R. HALMOS \\ HEINZ HOPF}

ALFRED HORN

\author{
R.D. JAMES \\ BØRGE JESSEN \\ PAUL LÉVY
}

GEORGE PÓLYA

J.J. STOKER

KOSAKU YOSIDA

\section{SPONSORS}

UNIVERSITY OF BRITISH COLUMBIA

UNIVERSITY OF SOUTHERN CALIFORNIA

CALIFORNIA INSTITUTE OF TECHNOLOGY

UNIVERSITY OF CALIFORNIA, BER KELEY

STANFORD RESEARCH INSTITUTE

STANFORD UNIVERSITY

UNIVERSITY OF CALIFORNIA, DAVIS

UNIVERSITY OF. UTAH

UNIVERSITY OF CALIFORNIA, LOS ANGELES

WASHINGTON STATE COLLEGE

UNIVERSITY OF CALIFORNIA, SANTA BARBARA

UNIVERSITY OF WASHINGTON

MONTANA STATE UNIVERSITY

UNIVERSITY OF NEVADA

OREGON STATE COLLEGE

AMERICAN MATHEMATICAL SOCIETY

HUGHES AIRCRAFT COMPANY

UNIVERSITY OF OREGON

SHELL DEVELOPMENT COMPANY

\section{UNIVERSTTY OF SOUTHERN CALIFORNIA}

Mathematical papers intended for publication in the Pacific Journal of Mathematics should be typewritten (double spaced), and the author should keep a complete copy. Manuscripts may be sent to any of the editors. Manuscripts intended for the outgoing editors should be sent to their successors. All other communications to the editors should be addressed to the managing editor, Alfred Horn, at the University of California Los Angeles 24, California.

50 reprints of each article are furnished free of charge; additional copies may be obtained at cost in multiples of 50 .

The Pacific Journal of Mathematics is published quarterly, in March, June, September, and December. The price per volume (4 numbers) is $\$ 12.00$; single issues, $\$ 3.50$; back numbers (Volumes $1,2,3$ ) are available at $\$ 2.50$ per copy. Special price to individual faculty members of supporting institutions and to individual members of the American Mathematical Society: $\$ 4.00$ per volume; single issues, $\$ 1.25$.

Subscriptions, orders for back numbers, and changes of address should be sent to the publishers, University of California Press, Berkeley 4, California.

Printed at Ann Arbor, Michigan. Entered as second class matter at the Post Office, Berkeley, California.

* During the absence of E.G. Straus.

UNIVERSITY OF CALIFORNIA PRESS - BERKELEY AND LOS ANGELES 


\section{Pacific Journal of Mathematics}

\section{Vol. 5, No. $3 \quad$ November, 1955}

Nesmith Cornett Ankeny and S. Chowla, On the divisibility of the class number of quadratic fields ............................. 321

Cecil Edmund Burgess, Collections and sequences of continua in the plane ........................................ 325

Jane Smiley Cronin Scanlon, The Dirichlet problem for nonlinear elliptic equations....................................... 335

Arieh Dvoretzky, A converse of Helly's theorem on convex sets ......... 345

Branko Grünbaum, On a theorem of L. A. Santaló................ 351

Moshe Shimrat, Simple proof of a theorem of P. Kirchberger .......... 361

Michael Oser Rabin, A note on Helly's theorem . ................... 363

Robert E. Edwards, On factor functions . . ................... 367

Robert E. Edwards, On certain algebras of measures ............... 379

Harley M. Flanders, Methods in affine connection theory.............. 391

Alfred Huber, The reflection principle for polyharmonic functions ........ 433

Geoffrey Stuart Stephen Ludford, Generalised Riemann invariants ....... 441

Ralph Gordon Selfridge, Generalized Walsh transforms............. 451 